EPJ Web of Conferences 53, 08014 (2013)

DOI: $10.1051 /$ epjconf/20135308014

(C) Owned by the authors, published by EDP Sciences, 2013

\title{
Development of new photon detection device for Cherenkov and fluorescence radiation
}

\author{
C. Aramo ${ }^{1}$, A. Ambrosio 2,3 , M. Ambrosio ${ }^{1}$, R. Battiston ${ }^{4}$, P. Castrucci ${ }^{5}$, \\ M. Cilmo ${ }^{1}$, M. De Crescenzi ${ }^{5}$, E. Fiandrini ${ }^{4}$, F. Guarino ${ }^{1,3}$, V. Grossi ${ }^{6}$, \\ P. Maddalena ${ }^{1,3}$, E. Nappi ${ }^{7}$, M. Passacantando ${ }^{6}$, G. Pignatel ${ }^{4}$, S. Santucci ${ }^{6}$, \\ M. Scarselli ${ }^{5}$, A. Tinti ${ }^{7}$ and A. Valentini ${ }^{7}$
}

${ }^{1}$ INFN, Sezione di Napoli, via Cintia 2, 80126 Napoli, Italy

${ }^{2}$ CNR-SPIN U.O.S. di Napoli

${ }^{3}$ Dipartimento di Scienze Fisiche, Università degli Studi di Napoli Federico II, via Cintia 2, 80126 Napoli, Italy

${ }^{4}$ INFN, Sezione di Perugia e Dipartimento di Fisica, Università degli Studi di Perugia, Piazza Università 1, 06100 Perugia, Italy

${ }^{5}$ Dipartimento di Fisica, Università degli Studi di Roma Tor Vergata, via della Ricerca Scientifica 1, 00133 Roma, Italy

${ }^{6}$ Dipartimento di Fisica, Università degli Studi dell'Aquila, via Vetoio 10, 67100 Coppito, L'Aquila, Italy

${ }^{7}$ INFN, Sezione di Bari e Dipartimento di Fisica, Università degli Studi di Bari, via Amendola 173, 70126 Bari, Italy

\begin{abstract}
Recent progress on the development of a new solid state detector allowed the use of finely pixelled photocathodes obtained from silicon semiconductors. SiPM detectors seem to be an ideal tool for the detection of Cherenkov and fluorescence light in spite of their not yet resolved criticism for operating temperature and intrinsic noise. The main disadvantage of SiPM in this case is the poor sensitivity in the wavelength range $300-400 \mathrm{~nm}$, where the Cherenkov light and fluorescence radiation are generated. We report on the possibility to realize a new kind of pixelled photodetector based on the use of silicon substrate with carbon nanotube compounds, more sensitive to the near UV radiation. Also if at the very beginning, the development of such detector appears very promising and useful for astroparticle physics, both in the ground based arrays and in the space experiments. The detectors are ready to be operated in conditions of measurements without signal amplification.
\end{abstract}

\section{INTRODUCTION}

Multiwall carbon nanotubes (MWCNTs) consist of multiple layers of graphite sheets forming concentric cylinders, from two to many tens. Some MWCNT properties made them an attractive material for UV photodetector [1,2] and photosensor applications, when associated their response to light stimulus. We present a novel large area photodetector featuring low noise, good efficiency in the wavelength range from 200 to $1000 \mathrm{~nm}$ and great surface uniformity. This detector has been obtained by coupling the optoelectronic characteristics of carbon nanostructures (CNs) with the well-known optoelectronic properties of silicon. The proposed device mimics the behaviour of a p-n-p phototransistor where the carbon nanostructure/silicon interface acts as the base, while the emitter is the Schottky

This is an Open Access article distributed under the terms of the Creative Commons Attribution License 2.0, which permits unrestricted use, distribution, and reproduction in any medium, provided the original work is properly cited. 

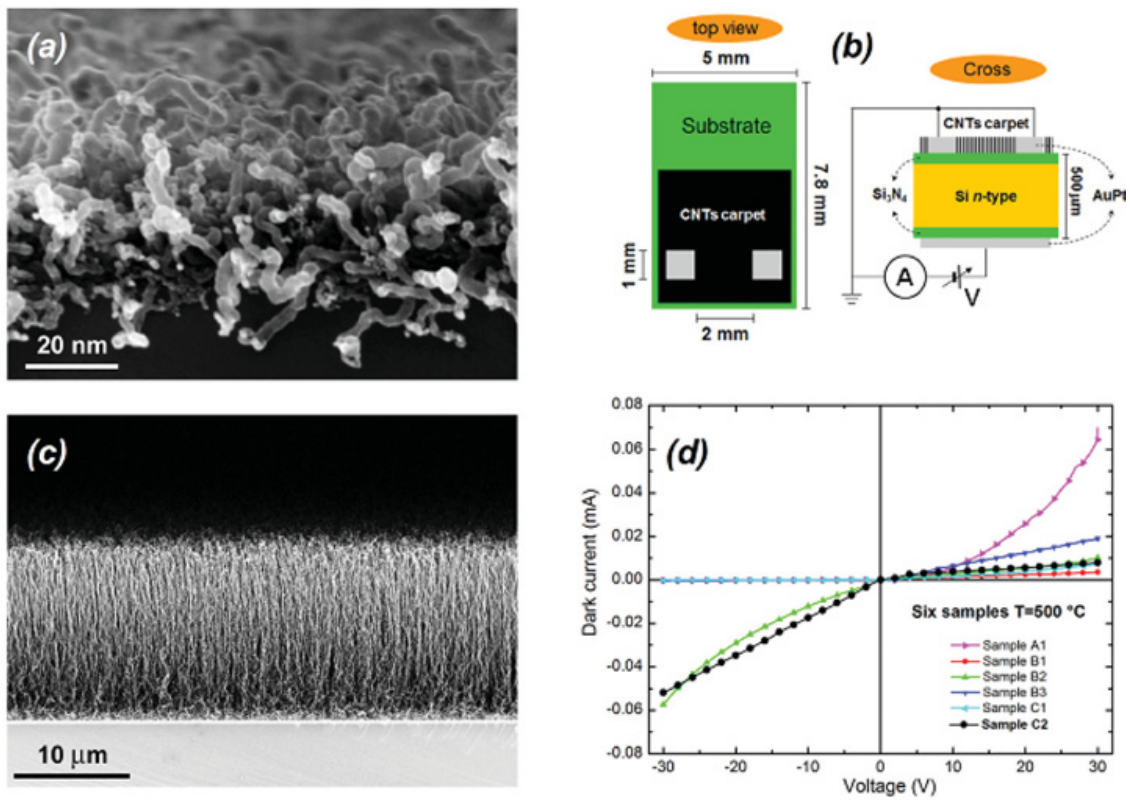

Figure 1. SEM images of $\mathrm{CN}$ grown at a temperature of $500^{\circ} \mathrm{C}$ (a) and $700{ }^{\circ} \mathrm{C}$ (c) on the substrate schematized in (b). See the morphological differences between carbon nanofibers (a) and the MWCNTs (c), induced by the growth temperature. (d), Dark current-voltage characteristic of six Si-CN devices.

Metal-Insulator-Semiconductor junction between the back electrode and the silicon substrate and the collector is the $\mathrm{CN}$ layer itself.

\section{CN-SI BASED DEVICE}

Figure 1a shows a SEM image of $\mathrm{CN}$ samples grown at a CVD temperature of $500{ }^{\circ} \mathrm{C}$, which behaves as bunches of ropes of carbon nanostructures with variable diameter along the tubes. By using a higher CVD growing temperature, $700^{\circ} \mathrm{C}$, (Fig. 1c) vertically aligned carbon nanotubes are obtained, with an average diameter of $19 \mathrm{~nm}$ and a length greater than 16 microns.

Figure 1c shows the schematic of the geometry of the device. CNs are grown in the black area around electrodes. As silicon substrate we used $500 \mathrm{~mm}$ thick n-doped silicon substrates $5 \times 7.8 \mathrm{~mm}^{2}$ sized with an average carrier concentration of $10^{14} \mathrm{~cm}^{-3}$, covered on both its faces by a $140 \mathrm{~nm}$ silicon nitride $\left(\mathrm{Si}_{3} \mathrm{~N}_{4}\right)$ layer. The drain voltage is applied between the electrodes of the top side and the electrode on the back (Fig. 1b) of the substrate. We first analysed the behaviour of six $500{ }^{\circ} \mathrm{C}$, whose dark current is reported in Fig. 1d. Most of them behave like a Schottky junction.

Figure $2 \mathrm{a}$ shows the photocurrent measured when the sample is illuminated with a $785 \mathrm{~nm}$ continuous laser beam at various light power intensities. The plot in Fig. 2a confirms the rectifying behaviour also in the presence of high intensity of illumination and in a large range of drain voltage. This is due to the particular structure of substrate in which the electrode on the back forms a Schottky MIS junction with the silicon substrate, whereas CNs form a carbon nanostructure-silicon heterojunction [3].

The device operates as a p-n-p phototransistor. As the applied voltage increases, the current is null until one reaches a few Volts threshold, then it increases linearly until saturation is reached. The breakdown limit has been observed well beyond a drain voltage of $-100 \mathrm{~V}$. On the other hand, as the light power intensity increases, a higher drain voltage is necessary to drain all the charges generated 
UHECR 2012
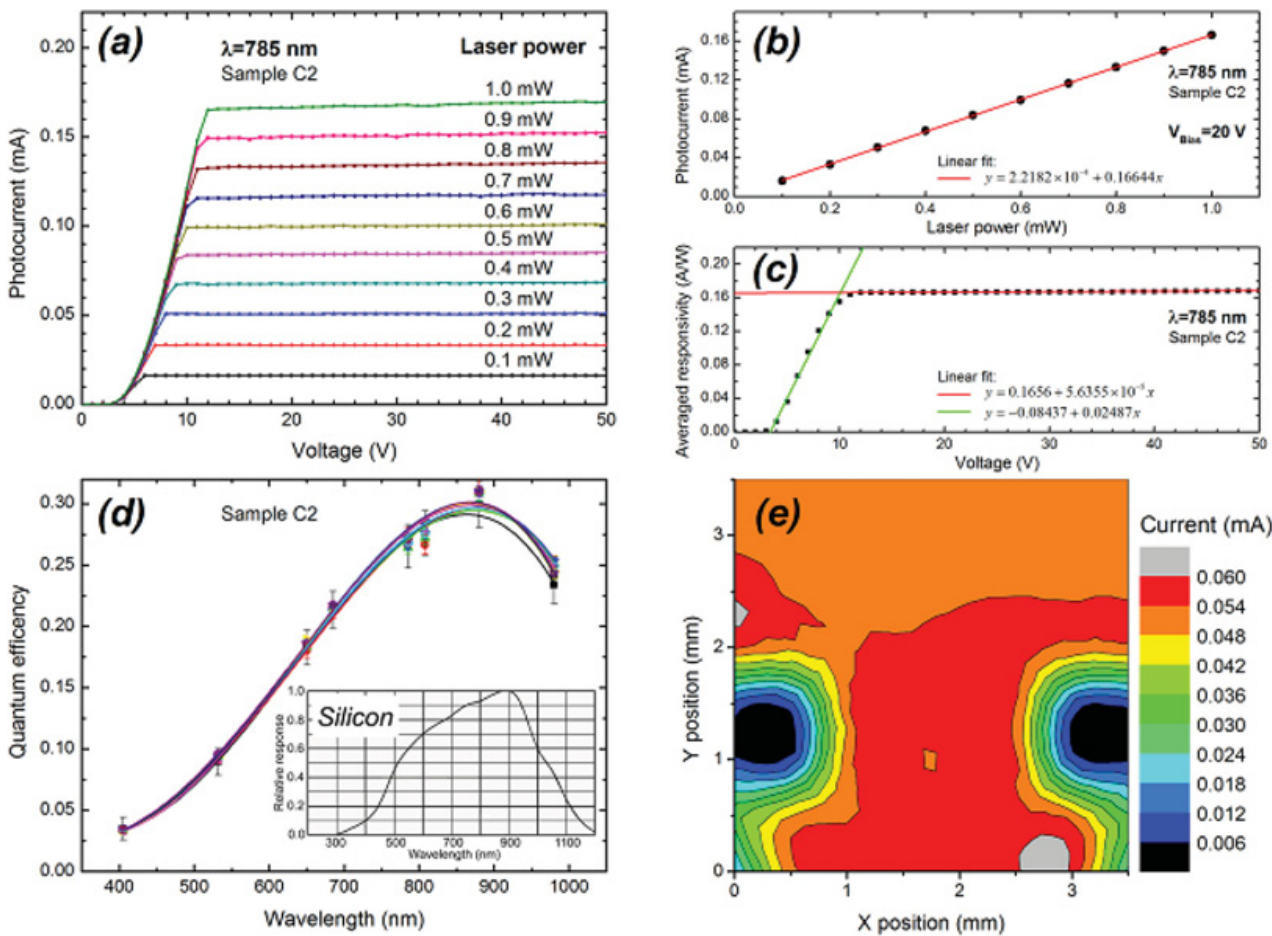

Figure 2. a) $\mathrm{I}-\mathrm{V}$ curve at $\lambda=785 \mathrm{~nm}$ for various laser light intensities. b) Linearity plot at $\lambda=785 \mathrm{~nm}$ for an applied drain voltage of $20 \mathrm{~V}$. c) Averaged responsivity at $\lambda=785 \mathrm{~nm}$. In the plot linear fit curves are superimposed fitting the signal rise and the current plateau. d) Quantum efficiency $(\mathrm{QE})$ as a function of the wavelength ranging from 405 to $980 \mathrm{~nm}$ fitted with a four order polynomial curve. In the inset the spectral response of silicon. e) Detector surface photocurrent map demonstrating the uniformity of sensitive area covered by CNs.

and reach the current saturation. It must be noted that no electronic amplification has been applied: the measured current is the one directly generated by photons impacting the $\mathrm{CN}$ layer.

\section{THE MWCNT-SI BASED DEVICE}

The same measurements have been repeated for the devices based on vertically aligned multiwall carbon nanotubes (Fig. 1c). The photosensitivity of the junction is shown in Fig. 3a reporting the I-V plot for device dark current and in Fig. $3 \mathrm{~b}$ reporting the photocurrent as a function of the drain voltage obtained using a $650 \mathrm{~nm}$ laser diode light at various power intensities. These I-V curves, as well as the average responsivity curve, show the same behaviour of the $\mathrm{CN}$-based device both as a function of the drain voltage and the laser light power intensity. However, the junction threshold increases significantly for MWCNTs reaching a value of $6.55 \mathrm{~V}$ (Fig. 3c), well higher than the $3.55 \mathrm{~V}$ thresholds measured for CNs (Fig. 2c). Moreover, the correlation between the detector quantum efficiency QE and the radiation wavelength is shown in Fig. 3d, fitted with a polynomial curve (blue curve) and compared with that of $\mathrm{CN}$-based device. In this case, the QE is four times higher than that measured for CN-based device in the UV energy region. The present result paves the road to the use of MWCNT-based detectors in the UV region. 

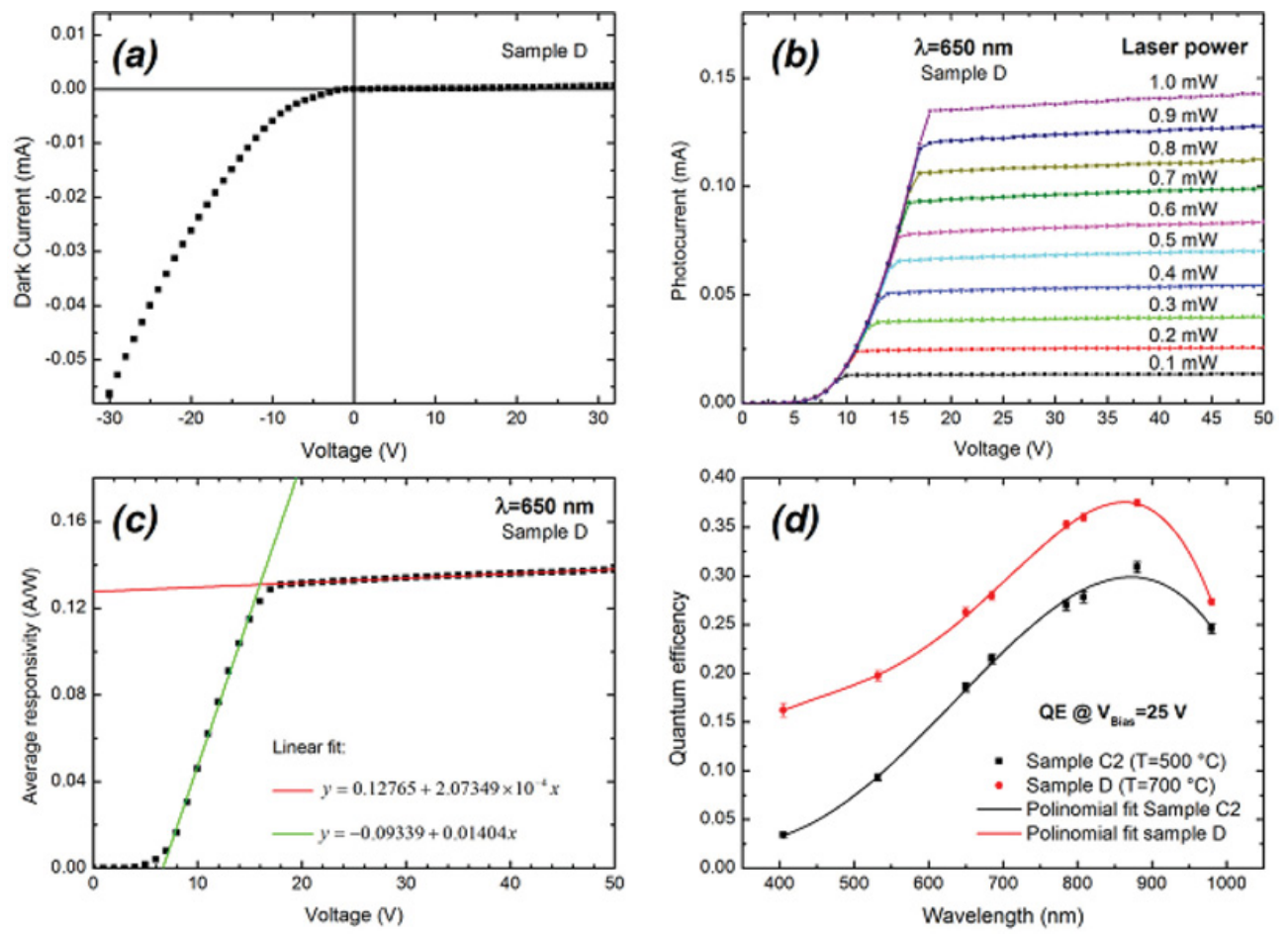

Figure 3. a) Dark current. b) $\mathrm{I}-\mathrm{V}$ curve at $\lambda=650 \mathrm{~nm}$ for various laser light intensities. c) Averaged responsivity at $\lambda=650 \mathrm{~nm}$. In the curves are superimposed fitting the signal rise and the current plateau. d) $Q E$ as a function of the wavelength ranging from 405 to $980 \mathrm{~nm}$ of detector @ $500^{\circ} \mathrm{C}$ compared with that @ $700^{\circ} \mathrm{C}$ fitted with a four order polynomial function.

\section{DISCUSSION}

The devices used for these measurements, in the absence of carbon nanostructures grown on their surface, show a backside MIS (Metal-Insulator-Semiconductor) junction between the n-doped silicon substrate and the metallic electrode [4]. An identical MIS junction between the silicon and the electrodes is present on the upper surface. Therefore, in the absence of CNTs the device can be schematized as a double Schottky junction polarized back-to-back (Fig. 4a). The presence of the CNTs overbalances the system: a heterojunction is created between the CNT layer and the silicon (Fig. 4b). The presence of the insulator (silicon nitrite) is bypassed by the formation of permanent low resistivity conduction channels inside this film, favouring the charge flow through the silicon substrate. The channels are created as an external voltage is applied to the device thanks to the peculiar characteristics of CNTs acting as one-dimensional conductive elements. In addition CNTs are semi-transparent to the radiation.

Thus light addressed on the CNT layer partially is converted in e-h pairs, partially reaches the silicon substrate in the depletion area under the CNT layer producing additional e-h pairs. The photo generated electrons or holes can migrate towards the CNT layer or can cross the junction from CNT to the silicon thanks to the presence of such low resistive ducts. The enhanced QE in the UV wavelength region is mainly due to photons converted inside the CNT layer while in the visible-near IR region light converts essentially inside the CNTs.

For this reason the device shows the characteristics of a p-n-p photo-transistor, where the metallic contact on the back acts as the emitter, the upper CNT layer is the collector and the CNT-silicon depletion area plays the role of the transistor base [5]. 
(a)

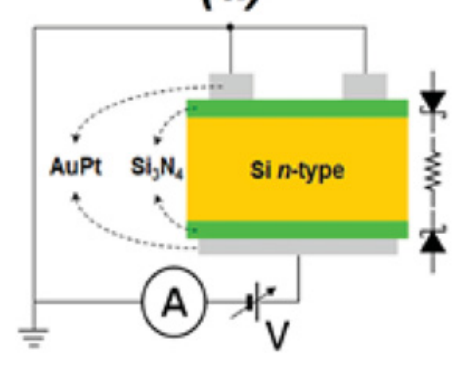

(b)

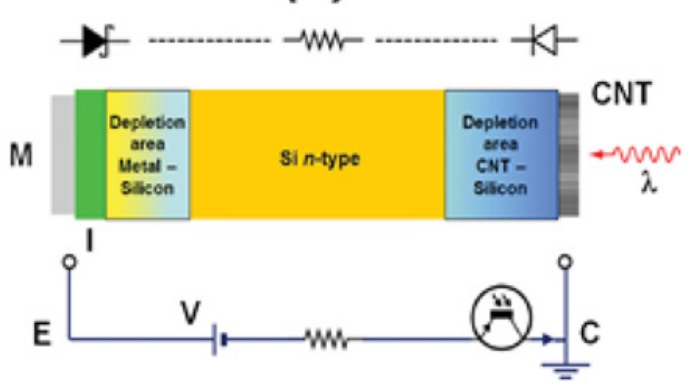

Figure 4. Layout of CNT-Si with the junctions. a) In absence of CNTs the device can be schematized as a double Schottky junction polarized back-to-back. b) The presence of the CNTs overbalances the system: a heterojuncion is created between the CNT layer and the silicon.

(a)

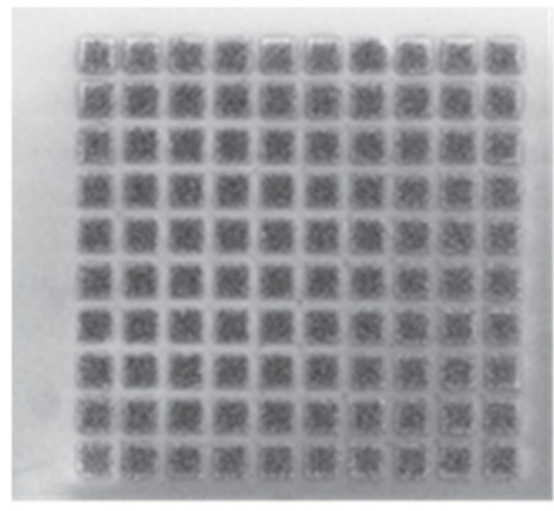

(b)

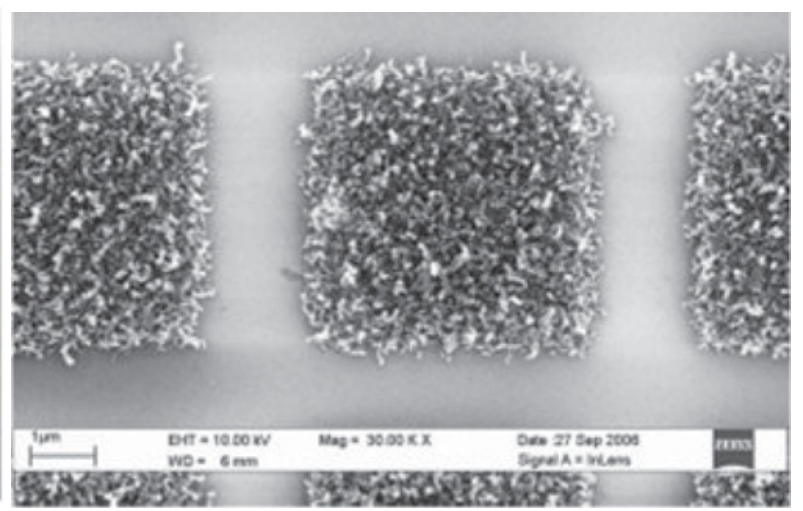

Figure 5. a) Pixels of $4 \times 4 \mu \mathrm{m}^{2}$ each obtained nanolithography. b) Individual CNT pixel.

\section{CONCLUSION}

The device is extremely promising in a future use in high energy physic experiments and medical applications. Highly pixelled surfaces can be obtained by means of micro or nano lithography (Fig. 5) [6] permitting the coupling of detector with external lighting devices such as scintillators and optical fibres. Our procedure to grow the carbon nanotubes with a definite pattern is based on a lift-off process. "Liftoff" is a method for making metallic patterns on a substrate, especially for those noble-metal thin films such as platinum, tantalum, nickel or iron, which are difficult to be etched by conventional methods [6]. For our device the dimension of pattern spreads from $10 \mu \mathrm{m}$ down to $100 \mathrm{~nm}$. This is obtained with an Electron Beam Lithography system. Figure 5a reports the results obtained with the combined use of nanolithography and lift-off process: a square matrix of $10 \times 10$ pixels, $4 \times 4 \mu \mathrm{m}^{2}$ each. What is important is that nanotubes grow only where the lift-off process left the catalyst: no carbon compounds appear to be present outside the pixel (Fig. 5b).

Pixelled large area photocathode can be used for the medical imaging or to manufacture fluorescence light detectors or to detect Cherenkov light cone with high accuracy. Progress on this line needs the cooperation of industry to develop single photon device by creating in the silicon base a secondary p-n junction acting as signal amplifier. 
The authors thank P. Di Meo, A. Pandalone e A. Vanzanella for their precious assistance for data acquisition and experimental instrumentation and B. Alfano for data acquisition programs.

A. has received funding from the FP7/ 2007-2013 under grant agreement N. 264098 - MAMA.

This work has been supported by Istituto Nazionale di Fisica Nucleare (INFN) in the frame of project SinPhoNIA (Single Photon Nanotechnology Innovative Approach).

\section{References}

[1] Ambrosio A. et al. NIM A 589, 398-403 (2008)

[2] Passacantando, M. et al. Appl. Phys. Lett. 93, 051911 (2008)

[3] Tinti A. et al. NIM A 629, 377-381 (2011)

[4] Ambrosio A. and Aramo C. (2011), ISBN: 978-953-307-496-2 InTech, http://www.intechopen com/articles/show/title/carbon-nanotubes-based radiation-detectors

[5] Ambrosio A. et al. JINST in press

[6] Ambrosio, M et al. NIM A 610, 1-10 (2009) 